\title{
Non-Linear Aperture for Stylized Depth of Field
}

Adrien Bousseau - INRIA / Grenoble University

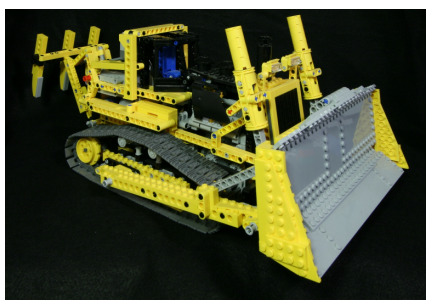

(a) Pinhole

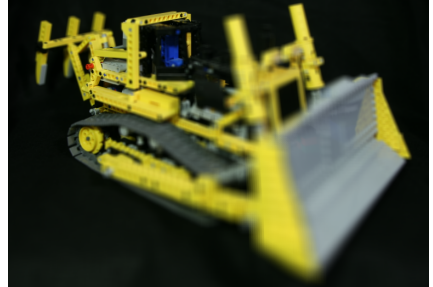

(b) Average

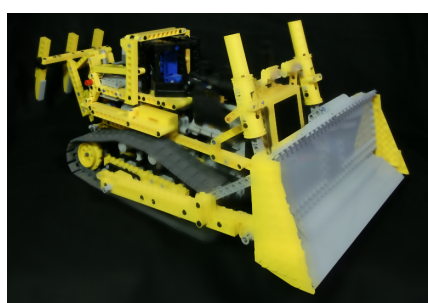

(c) Bilateral

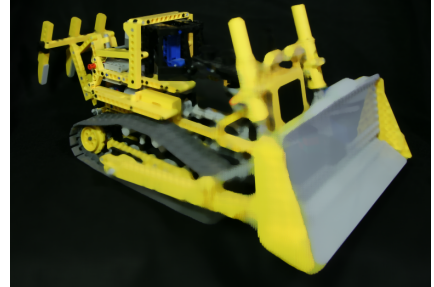

(d) Median

Figure 1: Compared to a traditional aperture that blurs out of focus points (b), non-linear apertures produce depth dependent non photorealistic simplifications. For example a bilateral filter smooths out details while preserving contours (c) and a median filter simplifies out of focus shapes $(d)$.

Abstract We introduce in this paper non-linear apertures that produce stylized depth of field from lightfield data. A non linear aperture is similar in spirit to the conventional aperture of a camera, except that it replaces the blur of shallow depth of field by a more complex detail removal filtering. The resulting non-realistic apertures produce stylized images where the amount of abstraction varies automatically with depth.

\section{Introduction and Related Work}

Shallow depth of field is widely used by photographers to blur the background and isolate the object of interest in a picture. On conventional cameras, the amount of blur of out of focus points of the scene is controled by the size of the aperture and the distance of the objects from the plane in focus. Lightfield cameras have been proposed to allow a user to vary the size of the aperture and the plane in focus after the shot [ $\mathrm{Ng}$ et al. 2005].

In the field of Non Photorealistic Rendering, similar attention grabing effects are obtained by removing the details of background objects with various abstraction filters. The use of different filters allows a greater variety of styles compared to the conventional out of focus blur of realistic photography. For instance, Winnemöller et al. [2006] applies a bilateral filter on images and videos to obtain a cartoon-like abstraction. However, in order to obtain depth dependent level of abstraction, a depth map is required. Such depth information can be painted by a user, which is tedious and not accurate, or estimated by vision algorithms, which is prone to errors.

We propose to combine abstraction and refocusing, and use nonlinear filters such as the bilateral filter in place of the average filter traditionally used in lightfield refocusing. Our approach is simple to integrate in existing lightfield viewers and allows depth dependant image abstraction without the need for explicit depth information.

\section{Linear and Non-Linear Apertures}

Box Filtering In traditional cameras, out of focus blur is produced by the optical integration of incoming light rays over the camera aperture. In lightfield refocusing, similar blur can be simulated by averaging the light rays over the synthetic aperture:

$$
p=\frac{1}{W} \sum_{u, v} L_{u, v, s, t}
$$

where $p$ is the resulting blurred pixel, $u$ and $v$ are the coordinates of the aperture samples, $s$ and $t$ are the coordinates of the intersection of the aperture rays with the focal plane, $L$ is the lightfield and $W$ is a normalization factor equal to the number of samples in the aperture. Figure $1(b)^{1}$ illustrates this realistic refocusing. In the following, we propose to modify the synthetic aperture equation to obtain varied stylization effects. We examplify here the median and bilateral filter.

Median Filtering The first non-linear aperture we propose consists in substituting the integration over the aperture by a median filter that selects the median value of the incoming light rays. Although such aperture could not be manufactured in reality, it can be simulated by the following synthetic aperture:

$$
p=\operatorname{median}_{u, v}\left\{L_{u, v, s, t}\right\}
$$

The resulting abstraction filter removes small details and simplifies shapes, as illustrated in Figure 1(d). Note that the amount of abstraction automatically increases with the distance to the plane in focus. This filter can be used as a basis for more complex stylization such as watercolor where the shape simplification mimics the use of a coarse paint brush.

Bilateral Filtering Finally, we propose to consider the aperture as the spatial support of a bilateral filter. The corresponding synthetic aperture can be expressed as:

$$
\begin{aligned}
p & =\frac{1}{W_{p}} \sum_{u, v} G_{\sigma_{r}}\left(\left|L_{u, v, s, t}-L_{u_{0}, v_{0}, s, t}\right|\right) L_{u, v, s, t} \\
\text { with } W_{p} & =\sum_{u, v} G_{\sigma_{r}}\left(\left|L_{u, v, s, t}-L_{u_{0}, v_{0}, s, t}\right|\right)
\end{aligned}
$$

where $G_{\sigma_{r}}$ is a gaussian function defining the range support, $u_{0}$ and $v_{0}$ are the coordinates of the central sample of the aperture. This filter removes the low contrast features of the image while preserving the strong edges (Figure 1(c)). The spatial support of the bilateral filter varies automatically with depth.

\section{Conclusion and Future Work}

We have presented in this paper a simple yet effective method to produce depth dependent stylized abstraction of photographs using lightfield data. We believe that similar lightfield stylization methods will be of great interest in the near future when lightfield cameras will become available to the average consumer.

\section{References}

NG, R., Levoy, M., Brédif, M., Duval, G., Horowitz, M., AND HANRAHAN, P. 2005. Light field photography with a hand-held plenoptic camera. Tech. rep.

Winnemöller, H., Olsen, S. C., AND GoOCh, B. 2006. Real-time video abstraction. ACM TOG (Proc. of SIGGRAPH 2006) 25, 3 .

\footnotetext{
${ }^{1}$ Lightfield data from http://lightfield.stanford.edu/
} 